\title{
ExpFit.nb: A MATHEMATICA program for the data processing of transverse relaxation times in nuclear magnetic resonance logging
}

\author{
Yoshinori WATANABE ${ }^{1}$ and Yoshito NAKASHIMA ${ }^{2}$
}

\begin{abstract}
Yoshinori Watanabe and Yoshito NAKAshima (2001) ExpFit.nb: A MATHEMATICA program for the data processing of transverse relaxation times in nuclear magnetic resonance logging. Bull. Geol. Surv. Japan, vol. 52 (6/7), p. 321-325, 6 figs, 1 table.
\end{abstract}

\section{Introduction}

The proton nuclear magnetic resonance (NMR) is a spectroscopy to observe the resonant transition of the hydrogen's nuclear spins between the Zeeman energy levels in a static magnetic field using radio-frequency waves. The hydrogen is a major element of groundwater and oil. Thus the proton NMR logging is a powerful method to estimate the porosity and permeability of water- and oil-bearing porous strata (e.g., Kleinberg, 1996; Nakashima, 2001). The advantages of proton NMR logging over conventional geophysical logging are (1) NMR signals from other elements (e.g., silicon) can be eliminated, so that signals derived only from the hydrogen-bearing substances (e.g., water molecules) are observable, (2) the pore-size distribution in porous rocks may be estimated by analyzing the transverse relaxation data, which cannot be accomplished by the neutron logging, and (3) quantitative scanning of the permeability of the watersaturated porous strata may be possible, which is difficult to obtain by other geophysical logging.

The raw data of the NMR logging are the timedomain spin-echo signals of the proton transverse relaxation obtained by the Carr-Purcell-Meiboom-Gill (CPMG) pulse sequence. Fitting the raw data with the linear combination of exponential functions is essential for the permeability- and porosity-estimate. The fitted coefficient of each exponential function should be non-negative because the coefficient is proportional to the population of hydrogen or water. This constraint makes the algorithm of the least-squares method complex (e.g., Minami, 1986). Although the least-squares program is essential to the interpreta-

${ }^{1}$ Center for Tsukuba Advanced Research Alliance, University of Tsukuba. Ten-nodai 1-1-1, Tsukuba, Ibaraki, 305-8577 Japan

${ }^{2}$ Institute for Geo-Resources and Environment, GSJ tion of the NMR logging, the computer programming is difficult and exhausting for most of geoscientists and there is no free program for the NMR data fitting as far as we know.

We developed a non-negative least-squares method program to fit the CPMG data with the linear combination of exponential functions. If CPMG data are given as a text file, the MATHEMATICA notebooktype program ExpFit.nb reads the data and outputs a text file including fitted coefficients. The program was applied successfully to synthetic CPMG data. We open the program to the public in order to facilitate the NMR study in the geophysical exploration.

\section{Mathematica Program}

The original free program ExpFit.nb is a notebooktype program for MATHEMATICA (http://www. wolfram.com/) version 4 . One can download the program at the authors' home pages (http://staff.aist.go. jp/nakashima.yoshito/progjp.htm, and http://staff. aist.go.jp/nakashima.yoshito/progeng.htm).

The CPMG time-series raw data, $x(t)$, are fitted with the linear combination of exponential functions:

$$
x(t)=c_{0}+\sum_{i=1}^{n} c_{i} \exp \left(-\frac{t}{\tau_{i}}\right)
$$

where $t$ is the time, $n$ is the number of the decay component, $\tau_{i}$ is the transverse relaxation time (T2), $c_{i}$ is the intensity of the contribution of the proton whose decay constant is $\tau_{i}$, and $c_{0}$ is the DC component of the noise (Kenyon, 1997). The coefficient, $c_{i}$, can be converted to the amount of the water if the calibration of the NMR signal is performed. The small $\tau_{i}$ corresponds to the small pores, and the large

Keywords: nuclear magnetic resonance logging, geophysical exploration, non-negative least-squares method, MATHEMATICA program, relaxation time 
$\tau_{i}$ is derived from the water in large ones. Thus it is possible to estimate the pore size distribution of water-filled porous media by the fitting.

The known quantities in Eq. 1 are $\tau_{i}(i=1,2,3, \ldots$, $n)$ and observation sets $(t, x(t))$; the unkown quantities are $c_{i}(i=1,2,3, \ldots, n)$ and $c_{0}$. Each preselected decay constant, $\tau_{i}$, is equally spaced on a logarithmic scale between $\tau_{1}$ and $\tau_{n}$ : $\log _{10} \tau_{i+1}-\log _{10} \tau_{i}=\left(\log _{10} \tau_{n}\right.$ $\left.-\log _{10} \tau_{1}\right) / k$ where $k=n-1$. Parameters $c_{i}$ and $c_{0}$ are determined by the least-squares method with the constraint that $c_{i}(i=1,2,3, \ldots, n)$ is non-negative. This constraint is necessary because the physical quantity, amount of water, is non-negative. The algorithm of the non-negative least-squares method is described by Lawson and Hanson (1974, p. 158-173).

Users are required to prepare a text file of the CPMG data named as cpmgdata.txt. There are two modes in running the program ExpFit.nb: one shows all calculation processes step by step on the computer display; the other displays only the final results of the least-squares calculation. The outputs from the program are (i) graphic display of cpmgdata.txt and a fitted curve and (ii) a text file results.txt in which the set $\left(\tau_{i}, c_{i}\right)$ is recorded. Numeric values in results.txt are separated not by $\mathrm{TAB}$ but by SPACE. It is straightforward to read results.txt using a commercially available spread-sheet software to draw a graph. The detail of the usage of the program is described within ExpFit.nb in Japanese.

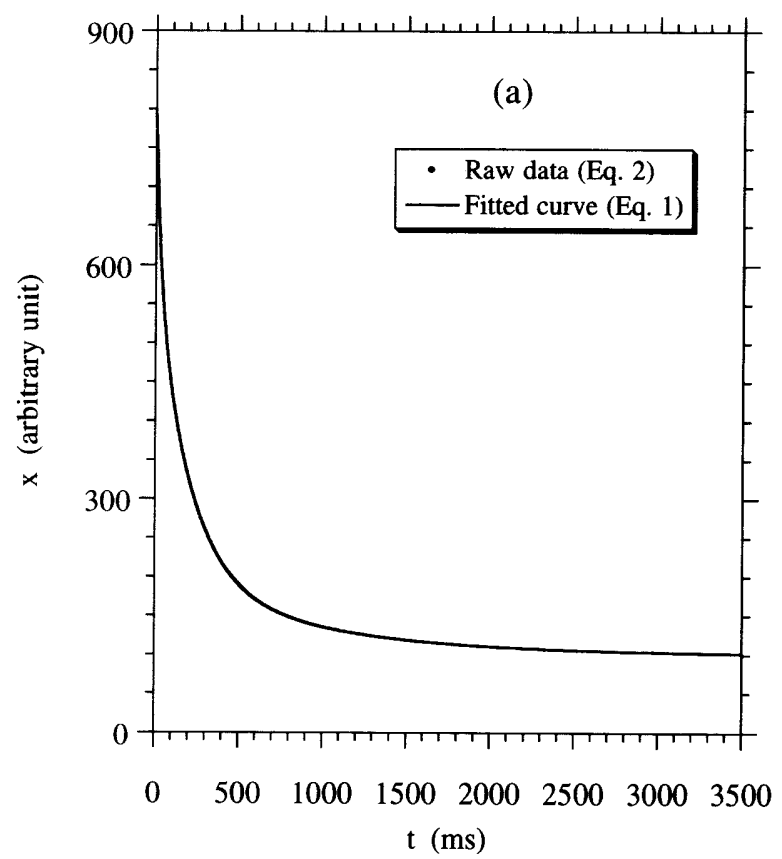

\section{Application to Synthetic NMR Data}

The program ExpFit.nb was applied to computergenerated NMR data to demonstrate the performance. We used the following synthetic signal as cpmgdata.txt:

$$
\begin{aligned}
x(t) & =200 \exp \left(-\frac{t}{30}\right)+400 \exp \left(-\frac{t}{200}\right) \\
& +100 \exp \left(-\frac{t}{900}\right)+g(m, s, t)
\end{aligned}
$$

where $t$ is in milliseconds and $g$ is a Gaussian noise with a mean of $m$ and standard deviation of $s$. The value of $m$ was taken to be 100 and the robustness of the program against the noise intensity $s$ was examined in the present study. The sampling interval or echo spacing is $1 \mathrm{~ms}$ and the number of the total data points is 3501 (i.e., $0 \leq t \leq 3500 \mathrm{~ms}$ ). The parameters of ExpFit.nb are $\tau_{1}=1 \mathrm{~ms}, \tau_{n}=10^{4} \mathrm{~ms}, n=41$, and $k$ $=40$. There is a trade-off between the execution time of the program and $n$ (the resolution of relaxation times). This is because the increase of $n$ requires longer CPU time to calculate, and $n=41$ is a conventional value for NMR logging (Kenyon, 1997). There are three exponential components in Eq. 2. As for the real data on NMR logging, the faster- (or slower-) decay component is derived from the protons in smaller (or larger) pores in water-saturated porous rocks. Hence in this example, the decomposition of the NMR signal into 3 components is essential to the distinction

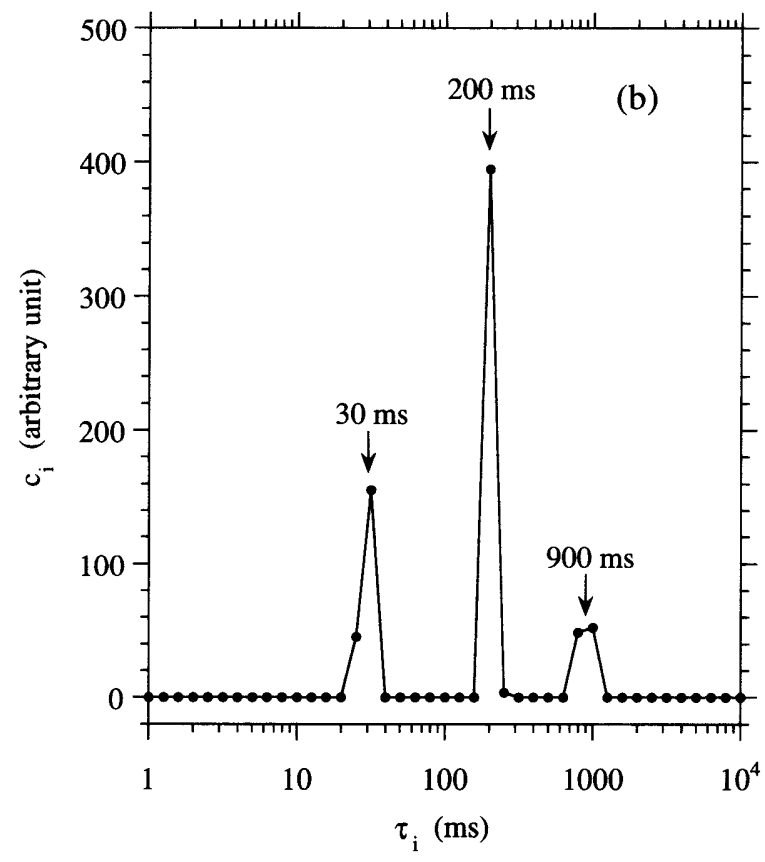

Fig. 1 Results of the application of ExpFit.nb to the synthetic NMR data of Eq. 2 without random noise (i.e., $g=g(100,0, t))$. (a) The graphic display of the cpmgdata.txt (Eq. 2) and fitted curve by Eq. 1 . The fit is so good, that it is hard to distinguish them. (b) Distribution of the relaxation times. The graphic display of the text file results.txt. The exact positions of the three decay-constants are indicated by arrows. 
Table 1 List of non-zero $c_{i}$ in Fig. 1(b). The fitted value of $c_{0}$ was 99.8517 which nearly equals the exact offset-value, $m=100$.

\begin{tabular}{ll}
\hline$\tau_{i}(\mathrm{~ms})$ & $c_{i}$ \\
\hline 25.1189 & 45.3181 \\
31.6228 & 155.406 \\
199.526 & 395.045 \\
251.189 & 3.63296 \\
794.328 & 48.8393 \\
1000 & 52.1433 \\
\hline
\end{tabular}

of the pore size. We expected the outputs of the program agreed to the prediction by Eq. 2 in terms of the decay-constants and amplitude of three exponential components.

The reliability of the program for the data without random noise was examined (Fig. 1). The input data (cpmgdata.txt, Eq. 2) for $g=g(100,0, t)$ and a fitted curve (Eq. 1) are shown by Fig. 1(a). This cpmgdata. txt is open at the authors' home pages so as to allow readers to trace easily the demonstration of Fig. 1. The execution time of the program was 23 minutes for Power Macintosh 7600/200 with a PowerPC 604e CPU $(200 \mathrm{MHz})$. The program output is the distribution or histogram of transverse relaxation time (T2) as shown in Fig. 1(b). There are 41 decay components (Fig. 1(b)), however, the number of decay components with non-

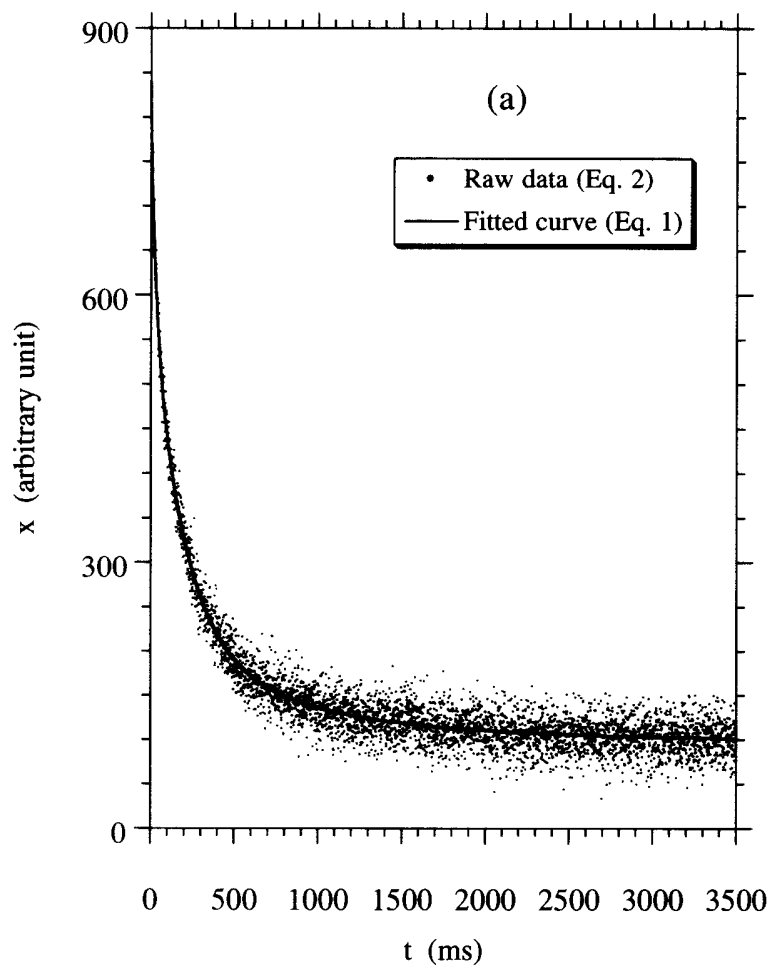

zero amplitude was 6 (Table 1). Figure 1(b) indicates the clear separation of three T2 components. Each peak is not very sharp but slightly spread and composed of two non-zero components. For example, the peak assigned to $30 \mathrm{~ms}$ is split into 25.1189 and 31.6228 ms. This is because the preselected 41 decayconstants do not include the exact value $(30 \mathrm{~ms})$ of the decay-constant of Eq. 2. Table 1 indicates that the weighted average of $\mathrm{T} 2$ for the peak around $30 \mathrm{~ms}$ is $(25.1189 \times 45.3181+31.6228 \times 155.406) /(45.3181+155.406)$ $\approx 30.2 \mathrm{~ms}$. The weighted averages of $\mathrm{T} 2$ for peaks around 200 and $900 \mathrm{~ms}$ are calculated to be about 200 . 0 and $900.5 \mathrm{~ms}$, respectively using Table 1 . Although there are slight discrepancy between the calculated values (30.2, 200.0, and $900.5 \mathrm{~ms})$ and exact ones (30, 200 , and $900 \mathrm{~ms}$ ), the three peak-positions nearly equal the exact values, which demonstrates the reliability of our program. The integrated intensities of peaks around 30,200 , and $900 \mathrm{~ms}$ in Table 1 are about 200.7, 398.7 , and 101.0 respectively. On the other hand, the exact values of the peak height are 200, 400, and 100 (Eq. 2). The integrated intensities are also nearly equal to the exact values. This agreement again demonstrates the reliable performance of ExpFit.nb.

The stability of our program against random noise was examined in Figs. 2 and 3. An example of CPMG data for $g=g(100,20, t)$ and its fitted curve are shown in Fig. 2(a); the distribution of transverse relaxation times is shown in Fig. 2(b). There is a ghost peak of

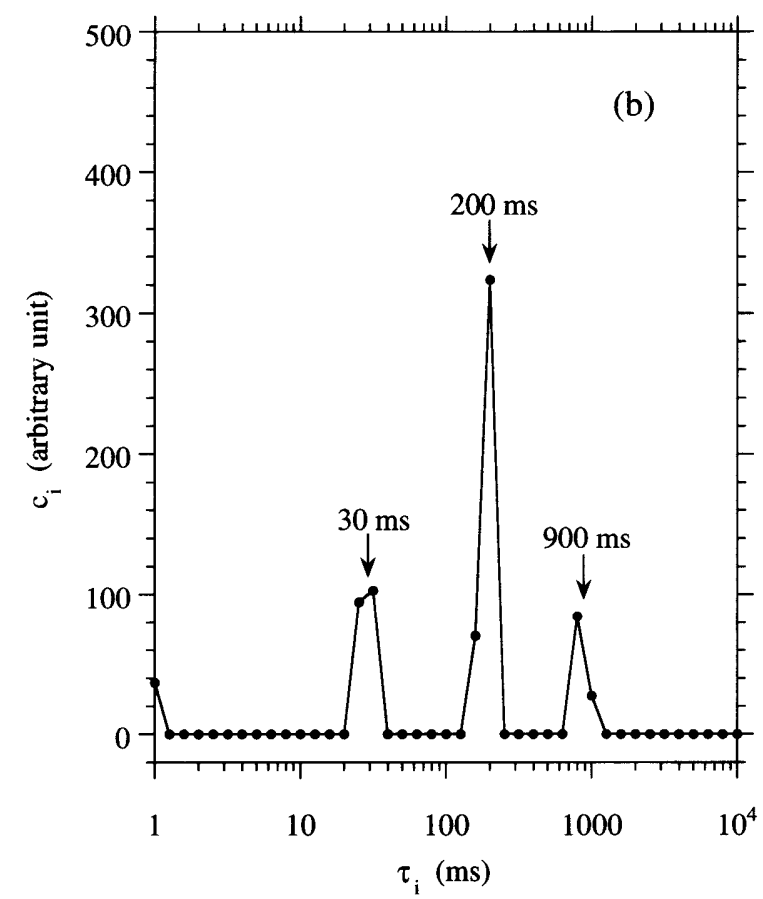

Fig. 2 Results of the application of ExpFit.nb to the NMR data of Eq. 2 with noise $g=g(100,20, t)$. (a) The graphic display of the NMR data and fitted curve by Eq. 1. (b) Distribution of the relaxation times. The exact positions of the three decay-constants are indicated by arrows. There is a noise-derived ghost peak at $1 \mathrm{~ms}$. 
the fast-decay component at $1 \mathrm{~ms}$ in Fig. 2(b). This is an undesirable effect of the random noise which has high-frequency components. The weighted $\mathrm{T} 2$ values of peaks around 30,200 , and $900 \mathrm{~ms}$ are calculated to be about 29,192 , and $845 \mathrm{~ms}$, respectively. The integrated intensity of each peak is about 197, 394, and 112. These results show again another undesirable effect of random noise which brings a discrepancy from the exact values.

The systematic simulation about the effects of noise intensity $s$ on the weighted T2 values (Fig. 3(a)) and on the integrated intensity (Fig. 3(b)) of each peak was performed. Ten sets of time-series data were generated for each of specific $s$ and the fitting by Eq. (1) was performed for each data set. Figure 3 illustrates that the discrepancy from the exact values increases with increasing $s$. This is an undesirable but inevitable effect of the random noise. The signal-to-noise ratio depends on the geological and geophysical conditions of wells. Hence the numerical simulation such as Fig. 3 should be performed using the signal-to-noise ratio of the wells in order to estimate the accuracy and resolution of the $\mathrm{T} 2$ distribution.

$\mathrm{T} 1$ (longitudinal relaxation time) analysis is as useful for the pore size estimation of porous rocks as $\mathrm{T} 2$ (transverse relaxation time) analysis (e.g., Davies et al., 1990). While the T2 raw data are decreasing time-series data (e.g., Fig. 1(a)), the signal of the T1

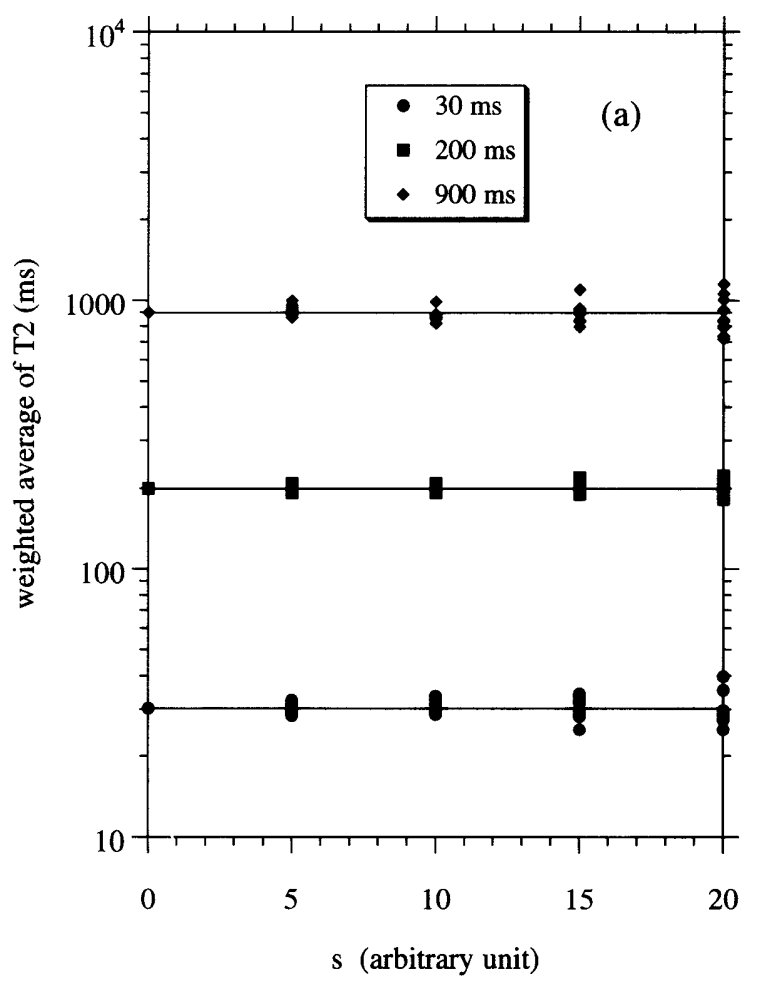

relaxation increases with time. Our program is applicable only to decaying signals because each exponential component in Eq. 1 decreases with time. However ExpFit.nb is applicable also to the T1 analysis by changing the sign of all the $\mathrm{T} 1$ relaxation data before the use of ExpFit.nb, for example, by multiplying ' -1 ' to the T1 signal. The non-negative leastsquares method is essential to the characterization of the pore structure of rocks by NMR. Our free program would contribute to the relaxation-data processing in NMR logging.

Acknowledgements: Comments by Dr. T. Nakatsuka were helpful. This study was supported by a grant from the Ministry of Economy, Trade and Industry of the Japanese government.

\section{References}

Davies, S., Kalam, M. Z., Packer, K. J. and Zelaya, F. O. (1990) Pore-size distributions from nuclear magnetic resonance spin-lattice relaxation measurements of fluid-saturated porous solids. II. Application to reservoir core samples. J. Appl. Phys., 67, 3171-3176.

Kenyon, W. E. (1997) Petrophysical principles of applications of NMR logging. The Log Analyst, 38, 21-43.

Kleinberg, R. L. (1996) Well logging. In Grant, D. M. and Harris, R. K. eds., Encyclopedia of

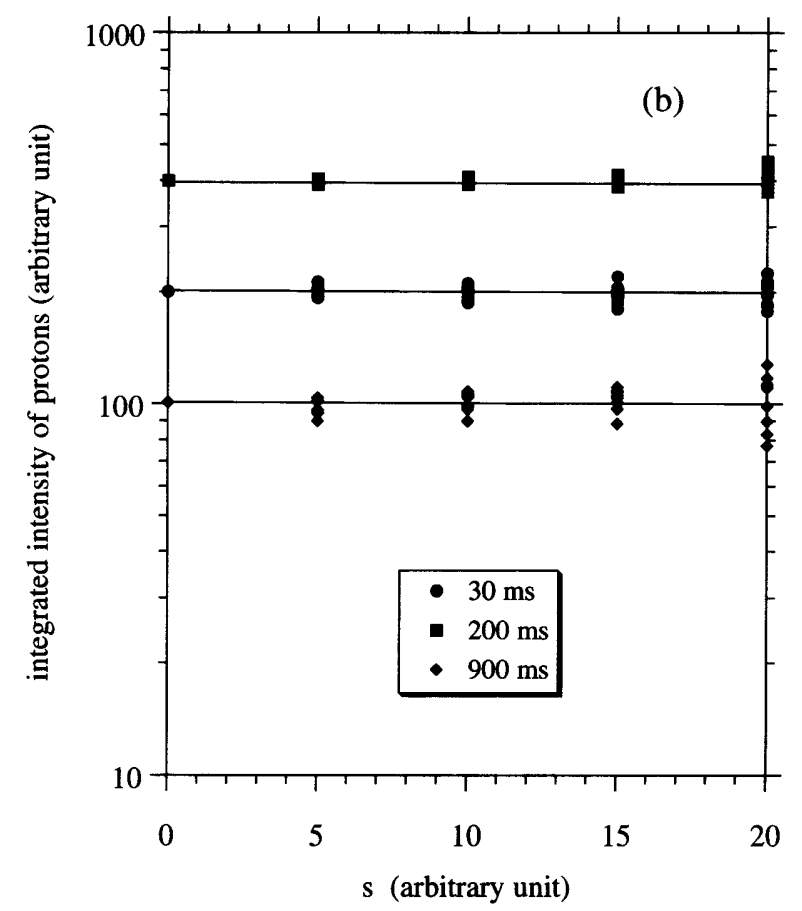

Fig. 3 The dependence of the three T2 components on the noise intensity, $s$. The number of fitting trials for each $s$ is 10 , and $m=100$. Exact values of $\tau_{i}$ and $c_{i}$ are indicated by horizontal lines. (a) Three T2 values depending on $s$. (b) Integrated intensities of the three decay components depending on $s$. 
Nuclear Magnetic Resonance, pp. 4960-4969, John Wiley \& Sons.

Lawson, C. L. and Hanson, R. J. (1974) Solving Least Squares Problems. Prentice-Hall, 340p. Minami, S. (1986) Wave Data Processing for the Scientific Measurements. CQ-Publishing Co., Ltd., 238p. (in Japanese).
Nakashima, Y. (2001) NMR logging applied to wells in geothermal zones, Sumikawa, Akita. J. Geotherm. Res. Soc. Japan, 23, 28-29. (in Japanese).

Received February 26, 2001

Accepted July 18, 2001 